\title{
Analytical Solutions of Eddy-Current Problems in a Finite Length Cylinder Yuriy Zhilichev ${ }^{1}$
}

\author{
${ }^{1}$ Durham, NC 27707 USA \\ *corresponding author, E-mail: yzhilichev@netscape. net
}

\begin{abstract}
Magnetic field and eddy currents in a cylinder of finite length are calculated by separation of variables. The magnetic field outside the cylinder or inside the bore of the hollow cylinder and shell is expressed in terms of Bessel functions. Both axial and transverse applied fields are considered for the solid and hollow cylinders. The equations for the vector potential components are transformed in one-dimensional equations along the radial coordinate with the consequent integration by the method of variation of parameters. The equation for the scalar electric potential when required is also integrated analytically. Expressions for the magnetic moment and loss are derived. An alternative analytical solution in terms of scalar magnetic potential is derived for the finite length thin shells. All formulas are validated by the comparison with the solutions by finite-element and finite-difference methods.
\end{abstract}

\section{Introduction}

The paper presents an analytical method of calculation of steady-state magnetic fields and eddy-currents in the cylinder of a finite length placed in the external axial or transverse magnetic field. It is known that for the infinitely long cylinder the closed form solutions were known in different forms [1-4]. However as it was pointed out by many authors $[1,2,5,6]$ that for the finite length cylinder the general analytical solution had not been available. For axial symmetry the distribution of eddy currents induced in a conducting rod of finite length by a coaxial coil is given in [7]. In general, the problem of a finite length cylinder earlier was solved by different numerical methods, and the solution was reported by several authors [6,8-11]. The conductive cylinder in transverse field was also included in so-called FELIX test problems in 80-ties [12-13] to verify the FEA codes. The analysis of eddy-currents in the cylinder is of interest for many practical applications such as electromagnetic shielding, designs of MRI /NMR components, conductive components of accelerator magnets, fusion reactors, induction heaters, turbogenerators and other electrical machines. The main aid of this paper is to derive a general analytical solution in terms of Bessel functions for eddy currents induced in a conductive cylinder by the quasi-static electromagnetic field.

\section{Formulation of the problem}

We consider the conductive non-magnetic cylinder with the constant electrical conductivity $\sigma$ and magnetic permeability $\mu_{0}$ placed in the applied magnetic field oscillating with the constant frequency $\omega$. The vector magnetic potential $A$ satisfies Amperes equation

$$
\operatorname{rotrot} \vec{A}=\mu_{0} \vec{j}
$$

where $\mu_{0}$ is the magnetic permeability of free space, $j$ is the current density for the area $r=\left[0, r_{0}\right], \phi=[0,2 \pi], z=\left[-z_{0}, z_{0}\right]$ and zero outside this region. The boundary condition at infinity

$$
\vec{A}(\infty)=\vec{A}_{0},
$$

where $A_{0}$ is the vector potential of applied magnetic field. From Ohm's law we can express the current density in terms of the vector and scalar $V$ potentials of electromagnetic field as:

$$
\vec{j}=-\sigma \frac{\partial \vec{A}}{\partial t}-\sigma \nabla V
$$

where $\sigma$ is the electrical conductivity of cylinder. The induced currents form the closed loops, and therefore current density satisfies the condition for the solenoidal field

$$
\operatorname{divj}=0 \text {. }
$$

The vector potential allows us to apply an additional gauging condition so that the field can be determined uniquely from the Maxwell equations. If we apply Coulomb gauge ( $\operatorname{div} \vec{A}=0$ ) equations (1), (4) become

$$
\begin{aligned}
& \nabla^{2} \vec{A}=-\mu_{0} \vec{j}, \\
& \nabla^{2} V=0
\end{aligned}
$$

with the coupled boundary conditions

$$
\frac{\partial V}{\partial n}_{\mid \Gamma}=-\frac{\partial \vec{A}}{\partial t} \vec{n}
$$

Equation (6) provides a zero component of eddy-currents normal to the surface $\Gamma$ of the conducive cylinder. The scalar potential is defined to be zero outside the conductor. After substitution (3) in (1a) we have a system of four differential equations that are coupled.

In orthogonal system of coordinates (1a) or (1b) can be solved by the method of separation of variables when the boundary $\Gamma$ coincides with the pieces of coordinates surfaces. A conductive cylinder falls in this category of geometries. However we have not found in literature the complete analytical solution for the eddy-current problem 
when the field is applied orthogonal to the cylinder axis. The major obstacle probably is in finding the analytical solution of the field in the whole space outside and inside the cylinder without subdividing the space in sub-regions. However in case of linear conductors there is no a rapid change of the flux density or field on its boundaries at least for moderate frequencies. Therefore the vector potential of magnetic field can be sought as a continuous smooth function in the whole space having piecewise conductive properties. The only problem seems to appear for the electric scalar potential that has to be coupled accurately with the vector magnetic potential on the boundaries of the conductor.

\section{Cylinder in axial field}

When cylinder is placed in the axial magnetic field (Fig.1) the problem can be significantly simplified. Only one component of vector potential is enough to formulate the problem. The scalar electric potential is not needed in this case. The complex azimuthal component of vector potential $A_{\phi} \exp (i \omega t)$ inside and outside the cylinder satisfies the equation

$$
\frac{\partial^{2} A_{\phi}}{\partial r^{2}}+\frac{1}{r} \frac{\partial A_{\phi}}{\partial r}-\frac{A_{\phi}}{r^{2}}+\frac{\partial^{2} A_{\phi}}{\partial z^{2}}=-\mu_{0} j_{\phi}
$$

and the far field boundary condition

$$
A_{\phi}(\infty)=B_{0} r / 2 \text {, }
$$

where $B_{0}$ is the amplitude of applied field. The vector potential of uniform field in form (2a) can be found by integrating the equation $\operatorname{rot} \vec{A}=\vec{B}[5,14]$.

The current density in (7) can be expressed in terms of the time derivative of vector potential

$$
i_{\phi}=-i \omega \sigma A_{\phi} .
$$

Since the magnetic permeability is the same through the whole space we can express the solution in the form of Fourier series accounting for the field symmetry as

$$
A_{\phi}=\sum_{m=1}^{\prime} A_{m}(r) \cos \lambda_{m} z
$$

In accordance with Grinberg method [15] after substituting (8) in (7) and integrating over the interval $\left[0, z_{\infty}\right]$ with the weight $\left(2 / z_{\infty}\right) \cos \lambda_{m} z$ we have

$$
\frac{\partial^{2} A_{m}}{\partial r^{2}}+\frac{1}{r} \frac{\partial A_{m}}{\partial r}-\frac{A_{m}}{r^{2}}-\lambda_{m}^{2} A_{m}=-\mu_{0} j_{m}
$$

where

$$
\begin{gathered}
j_{m}=-i \sigma \omega \sum_{l} d_{m l} A_{l}, \\
d_{m l}=\frac{2}{z_{\infty}} \int_{0}^{z_{0}} \cos \lambda_{m} z \cos \lambda_{l} z d z= \\
\frac{1}{z_{\infty}}\left(\frac{\sin \left(\lambda_{m}-\lambda_{l}\right) z_{0}}{\lambda_{m}-\lambda_{l}}+\frac{\sin \left(\lambda_{m}+\lambda_{l}\right) z_{0}}{\lambda_{m}+\lambda_{l}}\right) .
\end{gathered}
$$

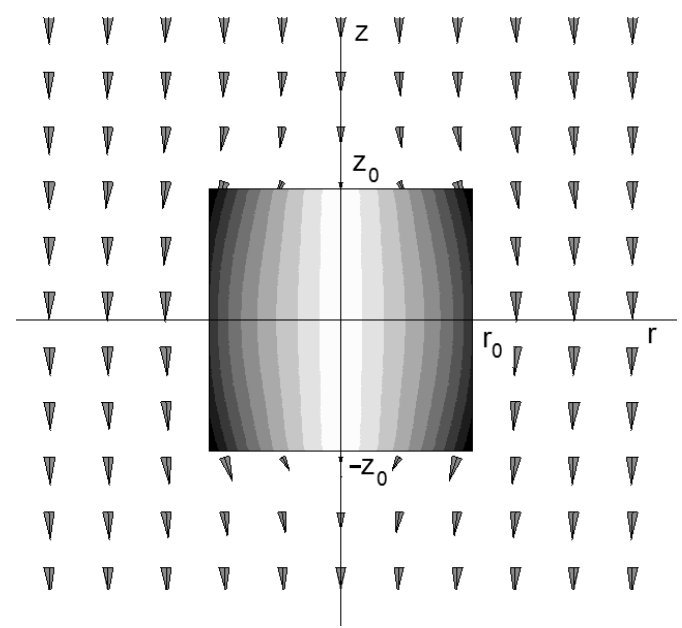

Figure 1: Conductive cylinder of radius $r_{0}$ and length $2 z_{0}$ in uniform axial field $B_{0}$ directed in $z$-axis.

where $\lambda_{m}=\pi(2 m-1) /\left(2 z_{\infty}\right), z_{\infty}$ is the axial coordinate where the field satisfies the far-field boundary condition (2a).

Using a method of variable parameters we can resolve (9) in the sum of a general solution of the homogeneous equation and a particular solution of (9)

$$
\begin{aligned}
& A_{m}(r)=\frac{B_{0} r d_{m 0}}{2}+C_{m} J_{1}\left(i \lambda_{m} r\right)+D_{m} H_{1}^{1}\left(i \lambda_{m} r\right)- \\
& \frac{\mu_{0} \pi}{2 i} J_{1}\left(i \lambda_{m} r\right) \int_{r}^{r_{0}} j_{m}\left(r^{\prime}\right) H_{1}^{1}\left(i \lambda_{m} r^{\prime}\right) r^{\prime} d r^{\prime} \\
& -\frac{\mu_{0} \pi}{2 i} H_{1}^{1}\left(i \lambda_{m} r\right) \int_{0}^{r} j_{m}\left(r^{\prime}\right) J_{1}\left(i \lambda_{m} r^{\prime}\right) r^{\prime} d r^{\prime}, m \geq 1
\end{aligned}
$$

where

$$
d_{m 0}=\frac{2}{z_{\infty}} \int_{0}^{z_{\infty}} \cos \lambda_{m} z d z=\frac{2}{\lambda_{m} z_{\infty}}(-1)^{m+1},
$$

$J_{1}$ and $H_{1}{ }^{1}$ are Bessel and Hankel functions, respectively, $C_{m}, D_{m}$ are the constants. To keep the potential finite at $r=0$, we have to eliminate the functions that have singularities on the cylinder axis. This is achieved by zeroing $D_{m}=0$ in (12). To satisfy the boundary condition (2a), we have to assume $C_{m}=0$ as well. Then, after replacing the functions of complex argument in (12) by the modified Bessel functions we rewrite equations for harmonics of vector potential as:

$$
\begin{aligned}
& A_{m}(r)=\frac{B_{0} r d_{m 0}}{2}+\mu_{0} I_{1}\left(\lambda_{m} r\right) \int_{r}^{r_{0}} j_{m}\left(r^{\prime}\right) K_{1}\left(\lambda_{m} r^{\prime}\right) r^{\prime} d r^{\prime}, \\
& +\mu_{0} K_{1}\left(\lambda_{m} r\right) \int_{0}^{r} j_{m}\left(r^{\prime}\right) I_{1}\left(\lambda_{m} r^{\prime}\right) r^{\prime} d r^{\prime}, m=1, \ldots M .
\end{aligned}
$$

After substitution $j_{m}$ using (10) and approximation of the integrals by quadratures on a regular grid $r_{n}=r_{n-1} h_{r n}$, $n=1, N$ in the interval $\left[0, r_{0}\right]$ one can derive a system of linear equations for the potential harmonics, $x_{k}=A_{m}\left(r_{n}\right)$, $k=n+(m-1) N$ :

$$
\sum_{k^{\prime}}^{\prime} c_{k k^{\prime}} \cdot x_{k^{\prime}}=f_{k},
$$

where 
$c_{k k^{\prime}}=\delta_{k k^{\prime}}+i \mu_{0} \sigma \omega h_{r n^{\prime}} r_{n^{\prime}} w_{n} w_{n^{\prime}} d_{m m^{\prime}}\left\{\begin{array}{l}I_{1}\left(\lambda_{m} r_{n^{\prime}}\right) K_{1}\left(\lambda_{m} r_{n}\right), n \geq n^{\prime} \\ I_{1}\left(\lambda_{m} r_{n}\right) K_{1}\left(\lambda_{m} r_{n^{\prime}}\right), n \leq n^{\prime}\end{array}\right\}$

$f_{k}=\frac{1}{2} B_{0} r_{n} d_{m 0}$,

$h_{\mathrm{r}}$ is the grid size over the radial coordinate, $\delta_{k k}$, is the Kronecker delta, $w_{n}$ are the quadrature weights. For the linear approximation of potential along the radial coordinate $w_{n}=1 / 2$ when $n=1, N$ and $w_{n}=1$ otherwise. A better accuracy is achieved when integrating the Bessel functions around each node of the grid analytically. For example, for internal nodes

$$
\begin{aligned}
& \int_{r_{n}-h_{r} / 2}^{r_{n}+h_{r} / 2} I_{1}\left(\lambda_{m} r^{\prime}\right) r^{\prime} d r^{\prime}=\frac{1}{\lambda_{m}^{2}}\left(\Psi\left[\lambda_{m}\left(r_{n}+h_{r} / 2\right)\right]-\Psi\left[\lambda_{m}\left(r_{n}-h_{r} / 2\right)\right]\right) \\
& \int_{r_{n}-h_{r} / 2}^{r_{n}+h_{r} / 2} K_{1}\left(\lambda_{m} r^{\prime}\right) r^{\prime} d r^{\prime}=\frac{1}{\lambda_{m}^{2}}\left(\Psi_{K}\left[\lambda_{m}\left(r_{n}+h_{r} / 2\right)\right]-\Psi_{K}\left[\lambda_{m}\left(r_{n}-h_{r} / 2\right)\right]\right)
\end{aligned}
$$

where $\Psi$ and $\Psi_{\mathrm{K}}$ are the combinations of modified Bessel and Struve functions defined in [16].

Formulas for harmonics of vector potential (14) can be easily modified for the hollow cylinder with the inner radius $r_{i}$. The only change required in (14) (that is now valid for $r \geq r_{i}$ ) is the replacement of zero by $r_{i}$ as a lower limit in all integrals over the radial coordinate. For hollow cylinders, it is of practical interest to calculate the field on the axis. The vector potential inside the bore $0 \leq r \leq r_{i}$

$$
A_{m}(r)=\frac{B_{0} r d_{m 0}}{2}+\mu_{0} I_{1}\left(\lambda_{m} r\right) \int_{r_{i}}^{r_{0}} j_{m}\left(r^{\prime}\right) K_{1}\left(\lambda_{m} r^{\prime}\right) r^{\prime} d r^{\prime} .
$$

The expression for the axial component can be derived as

$$
B_{z}=\sum_{m=1}^{M} B_{m}(0) \cos \lambda_{m} z
$$

where coefficients $B_{m}$

$B_{m}(0)=B_{0} d_{m 0}-i \mu_{0} \sigma \omega \lambda_{m} \int_{r_{i}}^{r_{0}} K_{1}\left(\lambda_{m} r\right) \sum_{l=1}^{M} d_{m l} A_{l}(r) r d r$

or after approximation the integral by the quadrature

$$
\begin{aligned}
& B_{m}(0)=B_{0} d_{m 0}-i \mu_{0} \sigma \omega \lambda_{m} \sum_{n=1}^{N} K_{1}\left(\lambda_{m} r_{n}\right) r_{n} w_{n} h_{r n} \sum_{l=1}^{M} d_{m l} A_{l}\left(r_{n}\right), \\
& r_{1}=r_{i}, r_{N}=r_{0} .
\end{aligned}
$$

For the field outside the cylinder the harmonics of potential are calculated in terms of harmonics on the grid within the cylinder as:

$$
\begin{aligned}
A_{m}\left(r>r_{0}\right)= & \frac{B_{0} r d_{m 0}}{2}-i \mu_{0} \sigma \omega K_{1}\left(\lambda_{m} r\right) \times, \\
& \sum_{n=1}^{N} I_{1}\left(\lambda_{m} r_{n}\right) r_{n} h_{r n} w_{n} \sum_{l=1}^{M} d_{m l} A_{l}\left(r_{n}\right)
\end{aligned}
$$

The radial component of flux density outside the cylinder $B_{r}\left(r>r_{0}\right)=\sum_{m=1}^{M} B_{m}\left(r>r_{0}\right) \sin \lambda_{m} z$

where

$B_{m}\left(r>r_{0}\right)=-i \mu_{0} \sigma \omega \lambda_{m} K_{1}\left(\lambda_{m} r\right) \sum_{n=1}^{N} I_{1}\left(\lambda_{m} r_{n}\right) r_{n} h_{r n} w_{n} \sum_{l=1}^{M} d_{m l} A_{l}\left(r_{n}\right)$,

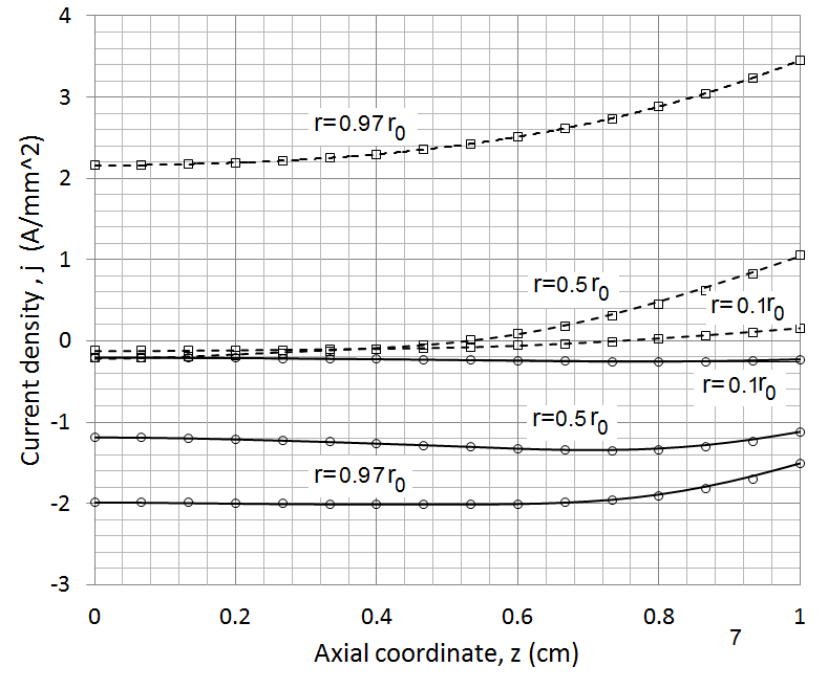

Figure 2: Current density across the cylinder of $r_{0}=1 \mathrm{~cm}$, $z_{0}=1 \mathrm{~cm}$ at $f=300 \mathrm{~Hz}, B_{0}=10 \mathrm{mT}$ : solid line $=$ real $j$, analytical , dash line $=$ imaginary $j$, analytical; o $=$ real $j$, FEA, $\square=$ imaginary $j$, FEA

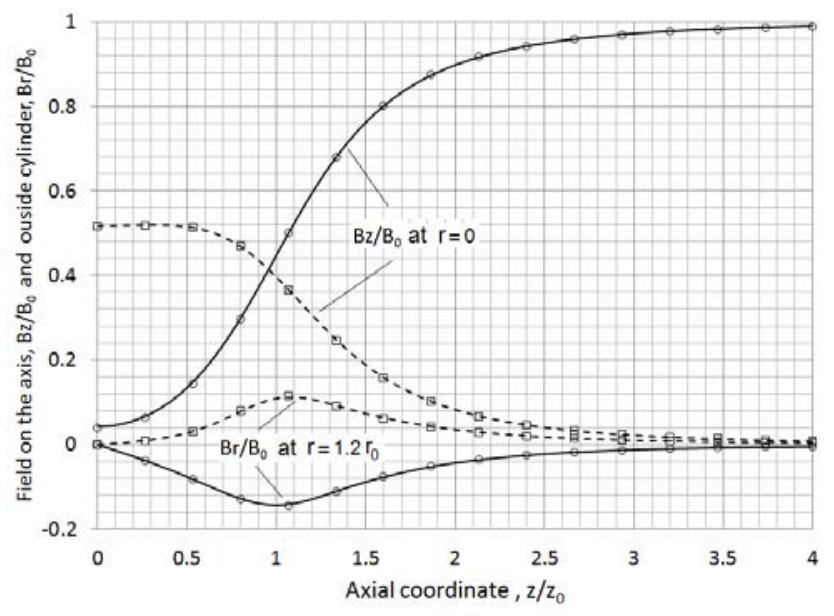

Figure 3: Relative flux density on the axis and outside of the hollow cylinder of $r_{i}=0.5 \mathrm{~cm}, r_{0}=1 \mathrm{~cm}, z_{0}=1 \mathrm{~cm}$ at $f=300$ $\mathrm{Hz}$ : solid line = real component , analytics, dash line =imaginary component, analytics; o = real component, FEA, $\square=$ imaginary component, FEA

The field inside has a similar structure but the modified Bessel functions of the first and second kind in (18) are swapped. The axial component of flux density can be derived as well. In general, it will include $K_{l}\left(\lambda_{m} r\right)$ and its derivative over the radial coordinate outside the cylinder and $I_{l}\left(\lambda_{m} r\right)$ and its derivative inside the cylinder if hollow.

For the thin cylindrical shells the number of steps over the radial coordinates can be significantly reduced. For just one step the set of equations (15) degrades to the system of equations for the potential harmonics at the same radial coordinate.

The total current induced in the cylinder is 


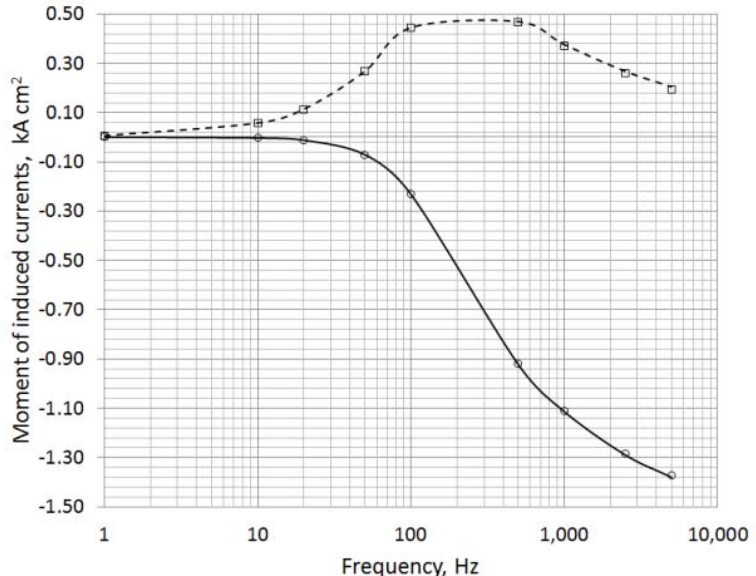

Figure 4: Moment of currents induced in the solid cylinder of $r_{0}=1 \mathrm{~cm}, z_{0}=1 \mathrm{~cm}$ at $B_{0}=10 \mathrm{mT}$ of axial field: solid line $=$ real component, analytics, dash line =imaginary component, analytical; $\mathrm{o}=$ real component, FEA, $\square=$ imaginary component, FEA.

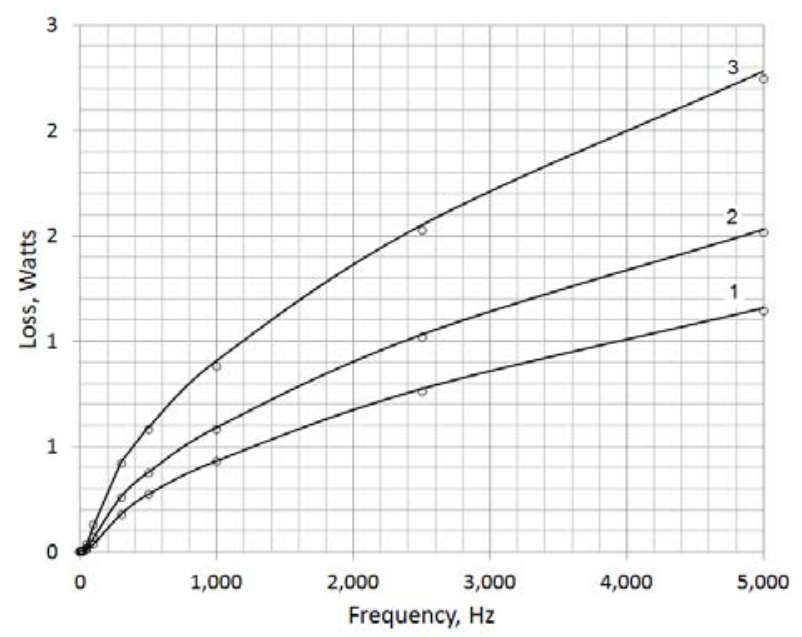

Figure 5: Power loss in the hollow cylinder of $r_{i}=0.5 \mathrm{~cm}$, $r_{0}=1 \mathrm{~cm}$ in the applied field of $B_{0}=10 \mathrm{mT}$ at different lengths, $2 z_{0}=1 \mathrm{~cm}(1) ; 2 \mathrm{~cm} \mathrm{(2),}=4 \mathrm{~cm} \mathrm{(3):} \mathrm{solid} \mathrm{line}=$ analytical solution $(21), \mathrm{o}=\mathrm{FEA}$.

$$
I_{\text {total }}=2 \int_{r_{i}}^{r_{0} \int_{0}^{z_{0}}} j_{\phi} d z d r=-2 i \sigma \omega \sum_{n=1}^{N} \sum_{m=1}^{M} \frac{w_{n} d_{r n}}{\lambda_{m}} A_{m}\left(r_{n}\right) \sin \lambda_{m} z_{0} .
$$

The moment of the induced currents is calculated as

$$
M_{z}=4 \pi \int_{r_{i}}^{r_{0} \int_{0}} \int_{\phi} j_{\phi}^{2} d z d r=-2 \pi i \sigma \omega \sum_{n=1}^{N} \sum_{m=1}^{M} \frac{w_{n} r_{n}^{2}}{\lambda_{m}} h_{r n} A_{m}\left(r_{n}\right) \sin \lambda_{m} z_{0}
$$

The power dissipated in the cylinder is

$$
P=\frac{2 \pi}{\sigma} \int_{r_{i}}^{r_{0} z_{0}} \int_{0} j_{\phi} j_{\phi}^{*} r d z d r=\frac{\pi z_{\infty}}{\sigma} \sum_{n=1}^{N} \sum_{m=1}^{M} \sum_{l=1}^{M} d_{m l} j_{m} j_{l}^{*} w_{n} r_{n} h_{r n} .
$$

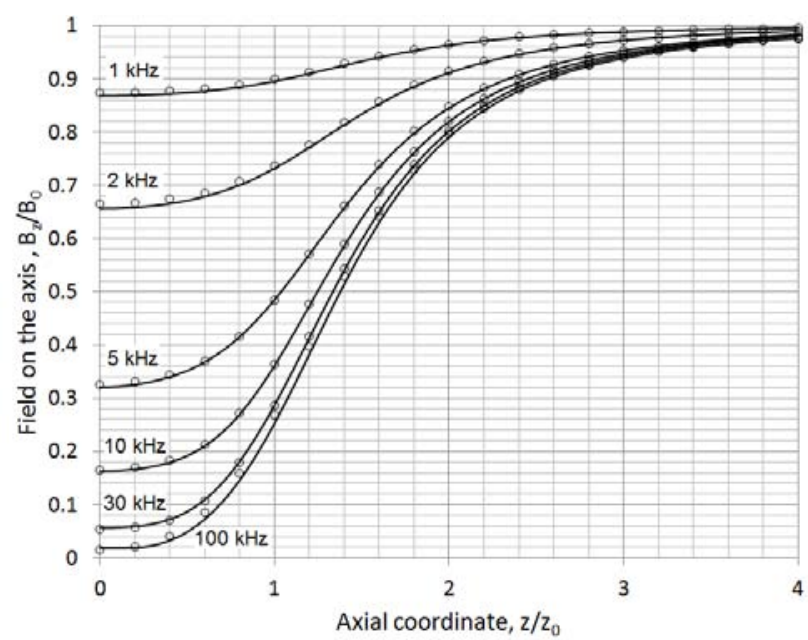

Figure 6: Relative amplitude of flux density on the axis of the thin cylindrical shell of $r_{0}=1 \mathrm{~cm}, z_{0}=1 \mathrm{~cm}$, thickness $h_{\mathrm{r}}=0.35 \mathrm{~mm}$ at different frequencies : solid line $=$ analytics, $\mathrm{o}=\mathrm{FEA}$.

Formulas for the field components, moment and power loss have been validated by the comparison with the results from FEA code [17]. The solid and hollow copper cylinders of radius $1 \mathrm{~cm}$ and various lengths have been considered. The results of comparison are presented in Fig.2-Fig.6. The number of harmonics $M$ in these numerical tests typically is in the range between 20 and 30, and number of steps in radial grid, $N$ is between 20 and 30 as well. The step $\Delta_{r n}$ depends on the frequency and it is selected based on the skin depth. The far field boundary (2) is applied at $z_{\text {inf }}$ that typically is selected in the range of $5 z_{0}$ or higher. To reduce the oscillations in the solution, Lanczos smoothing is applied in all series over the axial coordinate.

\section{Cylinder in transverse field}

\subsection{Solid cylinder}

Let's assume that the external field is applied in $x$-direction (Fig.7). The induced current density in the cylinder has radial, peripheral and axial components, so the problem becomes a 3D one. We denote the components of vector magnetic potential as $A_{r}, A_{\phi} A_{z}$, respectively. The potential satisfies (1a) in the whole space when Coulomb gauge is selected. The current density is non-zero for the area $r=[0$, $\left.r_{0}\right], \phi=[0,2 \pi], z=\left[-z_{0}, z_{0}\right]$, and is zero outside this region. The boundary condition at infinity [1], [3]

$$
A_{z}(\infty)=B_{0} r \sin \phi,
$$

Equation (1a) in the component notation can be written as

$$
\begin{aligned}
& \nabla^{2} A_{r}-\frac{A_{r}}{r^{2}}-\frac{2}{r^{2}} \frac{\partial A_{\phi}}{\partial \phi}=-\mu j_{r}, \\
& \nabla^{2} A_{\phi}-\frac{A_{\phi}}{r^{2}}+\frac{2}{r^{2}} \frac{\partial A_{r}}{\partial \phi}=-\mu j_{\phi}, \\
& \nabla^{2} A_{z}=-\mu j_{z},
\end{aligned}
$$


where

$$
\nabla^{2}=\frac{\partial^{2}}{\partial r^{2}}+\frac{1}{r} \frac{\partial}{\partial r}+\frac{1}{r^{2}} \frac{\partial^{2}}{\partial \phi^{2}}+\frac{\partial^{2}}{\partial z^{2}} .
$$

The scalar electric potential is also required to satisfy (5).

Because the magnetic field has a tangential symmetry over $x z$ - and $x y$-planes and normal symmetry over $z y$-plane the potential components can be presented as

$$
\begin{aligned}
& A_{r}=\mathrm{A}_{r}(r, z) \sin \phi, \quad \mathrm{A}_{r}(r, z)=\sum_{m=1} A_{r m}(r) \sin \lambda_{m} z, \\
& A_{\phi}=\mathrm{A}_{\phi}(r, z) \cos \phi, \quad \mathrm{A}_{\phi}(r, z)=\sum_{m=1} A_{\phi m}(r) \sin \lambda_{m} z, \\
& A_{z}=\mathrm{A}_{z}(r, z) \sin \phi, \quad \mathrm{A}_{z}(r, z)=\sum_{m=1} A_{z m}(r) \cos \lambda_{m} z,
\end{aligned}
$$

where $\lambda_{m}=\pi(2 m-1) /\left(2 z_{\infty}\right)$.

Since the cylinder has a finite length of $L=2 z_{0}$ the current density and electric scalar potential are zero beyond its volume while the magnetic field should be extended in $z$ direction far enough from the cylinder top and base faces. We assume that the magnetic field is equal to the applied field at $z_{\infty} \leq z \geq z_{\infty}$. Thus the current density and electric potential are expanded in the Fourier series over the interval $z=\left[-z_{0}, z_{0}\right]$ while the vector potential is fit in the interval $z=[-$ $\left.z_{\infty}, z_{\infty}\right]$.For the current density and electric scalar potential in the cylinder, we also account for the model symmetry

$$
\begin{aligned}
& j_{r}=\mathfrak{I}_{r} \sin \phi, \quad \mathfrak{I}_{r}=\sum_{l=1} j_{r l}(r) \sin \eta_{l} z, \\
& j_{\phi}=\mathfrak{I}_{\phi} \cos \phi, \quad \mathfrak{I}_{\phi}=\sum_{l=1} j_{\phi l}(r) \sin \eta_{l} z, \\
& j_{z}=\mathfrak{I}_{z} \sin \phi, \quad \mathfrak{I}_{z}=\sum_{l=1} j_{z l}(r) \cos \eta_{l} z, \\
& V=v \sin \phi, \quad v=\sum_{k=1} V_{k}(r) \sin \eta_{k} z,
\end{aligned}
$$

where $\eta_{\mathrm{k}}=\pi(2 k-1) /\left(2 z_{0}\right)$.

Equation (1e) contains only one component of vector potential and can be solved similar to (7). After substitution series (24) in (1e) and integration over $\left[-z_{\infty}, z_{\infty}\right]$ the equation for the potential harmonics is

$$
\frac{\partial^{2} A_{z m}}{\partial r^{2}}+\frac{1}{r} \frac{\partial A_{z m}}{\partial r}-\frac{A_{z m}}{r^{2}}-\lambda_{m}^{2} A_{z m}=-\mu_{0} \widetilde{j}_{z m}
$$

where

$$
\begin{gathered}
\widetilde{j}_{z m}=\sum_{l} a_{m l} j_{z l}, \\
a_{m l}=\frac{1}{z_{\infty}} \int_{-z_{0}}^{z_{0}} \cos \lambda_{m} z \cos \eta_{l} z d z= \\
\frac{(-1)^{l}}{z_{\infty}} \frac{2 \eta_{l}}{\lambda_{m}^{2}-\eta_{l}^{2}} \cos \frac{\pi(2 m-1) z_{0}}{2 z_{\infty}}, \quad m, l \geq 1 .
\end{gathered}
$$

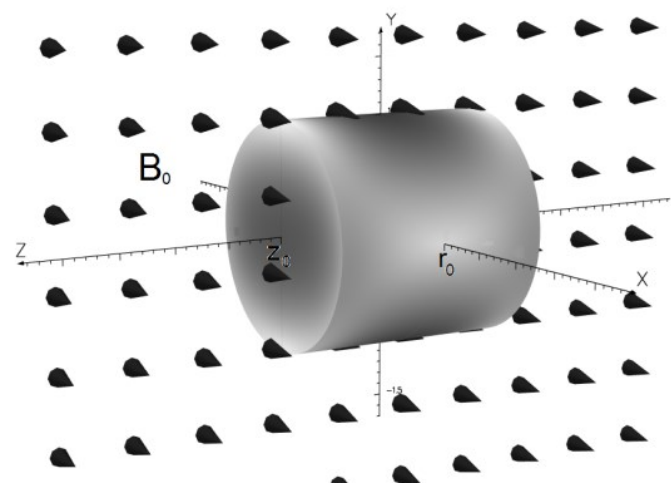

Figure 7: Conductive cylinder of radius $r_{0}$ and axial length $2 z_{0}$ in uniform transverse field directed in $x$-axis

Similarly to the solution of (9) we can resolve (29) in the sum of a general solution of the homogeneous equation and a particular solution. Because the current density harmonics (30) is dependent on the radial coordinate we use the method of variable parameters to express the harmonics $A_{z m}$ in the form of integrals

$$
\begin{aligned}
& A_{z m}=-B_{0} r d_{m 0}+\mu_{0} I_{1}\left(\lambda_{m} r\right) \int_{r}^{r_{0}} \widetilde{j}_{z m}\left(r^{\prime}\right) K_{1}\left(\lambda_{m} r^{\prime}\right) r^{\prime} d r^{\prime}, \\
& +\mu_{0} K_{1}\left(\lambda_{m} r\right) \int_{0}^{r} \widetilde{j}_{z m}\left(r^{\prime}\right) I_{1}\left(\lambda_{m} r^{\prime}\right) r^{\prime} d r^{\prime}, \quad \mathrm{m} \geq 1 .
\end{aligned}
$$

Equations (1c) and (1d) for the radial and angular components of vector potential contain the crossing terms. To avoid coupling between the equations, few manipulations on (1c) and (1d) should be performed. First, let's exclude the polar angle from the consideration by substituting $A_{r}, A_{\phi}$ into (1c) and (1d) in accordance with (22)-(23), respectively

$$
\begin{aligned}
& \Delta \mathrm{A}_{r}-\frac{2 \mathrm{~A}_{r}}{r^{2}}+\frac{2 \mathrm{~A}_{\phi}}{r^{2}}=-\mu_{0} \mathfrak{J}_{r}, \\
& \Delta \mathrm{A}_{\phi}-\frac{2 \mathrm{~A}_{\phi}}{r^{2}}+\frac{2 \mathrm{~A}_{r}}{r^{2}}=-\mu_{0} \mathfrak{J}_{\phi},
\end{aligned}
$$

where

$$
\Delta=\frac{\partial^{2}}{\partial r^{2}}+\frac{1}{r} \frac{\partial}{\partial r}+\frac{\partial^{2}}{\partial z^{2}}
$$

Next, we combine (33) and (34), namely we add and subtract them from each other with obtaining the equations for the new variables $A_{\phi+r}=A_{\phi}+A_{r}$ and $A_{\phi-r}=A_{\phi}-A_{r}$

$$
\begin{aligned}
& \Delta \mathrm{A}_{\phi+r}=-\mu_{0}\left(\mathfrak{I}_{\phi}+\mathfrak{J}_{r}\right), \\
& \Delta \mathrm{A}_{\phi-r}-\frac{4 \mathrm{~A}_{\phi-r}}{r^{2}}=-\mu_{0}\left(\mathfrak{I}_{\phi}-\mathfrak{I}_{r}\right) .
\end{aligned}
$$

Note that the variables $A_{\phi+r}$ and $A_{\phi-r}$ in (35) and (36) are decoupled now, and the solutions can be expressed in terms of Bessel functions of integer index. After multiplying (35) and (36) by $1 / z_{\infty} \sin \lambda_{m} z$ and integrating over $\left[-z_{\infty}, z_{\infty}\right]$ the equations for the harmonics are 


$$
\begin{aligned}
& \frac{\partial^{2} A_{\phi+r, m}}{\partial r^{2}}+\frac{1}{r} \frac{\partial A_{\phi+r, m}}{\partial r}-\lambda_{m}^{2} A_{\phi+r, m}=-\mu_{0}\left(\widetilde{j}_{r m}+\widetilde{j}_{\phi m}\right) \\
& \frac{\partial^{2} A_{\phi-r, m}}{\partial r^{2}}+\frac{1}{r} \frac{\partial A_{\phi-r, m}}{\partial r}-\frac{4 A_{\phi-r, m}}{r^{2}}-\lambda_{m}^{2} A_{\phi-r, m}=-\mu_{0}\left(\widetilde{j}_{\phi m}-\widetilde{j}_{r m}\right) .
\end{aligned}
$$

where

$A_{\phi \pm r, m}=A_{\phi, m} \pm A_{\phi-r, m}$,

$\widetilde{j}_{\phi m}=\sum_{l} b_{m l} j_{\phi l}$,

$\widetilde{j}_{r m}=\sum_{l} b_{m l} j_{r l}$

$b_{m l}=\frac{1}{z_{\infty}} \int_{-z_{0}}^{z_{0}} \sin \lambda_{m} z \sin \eta_{l} z d z=\frac{(-1)^{l}}{z_{\infty}} \frac{2 \lambda_{m}}{\lambda_{m}^{2}-\eta_{l}^{2}} \cos \frac{\pi(2 m-1) z_{0}}{2 z_{\infty}}, \quad m, l \geq 1$

The solution of one-dimensional equations (37) and (38) can be written in terms of Bessel functions similar to solution of (32)

$$
\begin{aligned}
& A_{\phi+r, m}=\mu_{0} I_{0}\left(\lambda_{m} r\right) \int_{r}^{r_{0}}\left(\widetilde{j}_{\phi m}\left(r^{\prime}\right)+\left(\widetilde{j}_{r m}\left(r^{\prime}\right)\right) K_{0}\left(\lambda_{m} r^{\prime}\right) r^{\prime} d r^{\prime},\right. \\
& +\mu_{0} K_{0}\left(\lambda_{m} r\right) \int_{0}^{r}\left(\widetilde{j}_{\phi m}\left(r^{\prime}\right)+\left(\widetilde{j}_{r m}\left(r^{\prime}\right)\right) I_{0}\left(\lambda_{m} r^{\prime}\right) r^{\prime} d r^{\prime}, \quad \mathrm{m} \geq 1\right. \\
& A_{\phi-r, m}=\mu_{0} I_{2}\left(\lambda_{m} r\right) \int_{r}^{r_{0}}\left(\widetilde{j}_{\phi m}\left(r^{\prime}\right)-\left(\widetilde{j}_{r m}\left(r^{\prime}\right)\right) K_{2}\left(\lambda_{m} r^{\prime}\right) r^{\prime} d r^{\prime},\right. \\
& +\mu_{0} K_{2}\left(\lambda_{m} r\right) \int_{0}^{r}\left(\widetilde{j}_{\phi m}\left(r^{\prime}\right)-\left(\widetilde{j}_{r m}\left(r^{\prime}\right)\right) I_{2}\left(\lambda_{m} r^{\prime}\right) r^{\prime} d r^{\prime}, \quad \mathrm{m} \geq 1\right.
\end{aligned}
$$

The current density harmonics $j_{r m}, j_{\phi m}, j_{z m}$ in (32),(39), and (40) can be determined from the condition for the divergence-free current density (4). Let's impose the Coulomb gauge for the vector potential. Then the scalar electric potential satisfies the Laplace equation (5). After substituting (28) in (5), multiplying it by $1 / z_{0} \sin \eta_{k} z$ and integrating over $\left[-z_{0}, z_{0}\right]$ the equations for the harmonics of scalar potential are

$$
\frac{\partial^{2} V_{k}}{\partial r^{2}}+\frac{1}{r} \frac{\partial V_{k}}{\partial r}-\frac{V_{k}}{r^{2}}-\eta_{k}^{2} V_{k}=q_{k},
$$

where the right part in (41) appears because the normal derivative of scalar potential on the cylinder surface at $z= \pm$ $z_{0}$ is different from zero. It can be further expressed through the time derivative of vector potential in accordance with (6)

$$
q_{k}=-\left.\frac{2(-1)^{k+1}}{z_{0}} \frac{\partial v}{\partial z}\right|_{z=z_{0}}=(-1)^{k} \frac{2 i \omega}{z_{0}} \mathrm{~A}_{z}\left(z_{0}\right) .
$$

The boundary conditions for the harmonics of scalar potential are

$$
\begin{aligned}
& V_{k}(0)=0, \\
& {\frac{\partial V_{k}}{\partial r}}_{\mid r=r_{0}}=-i \omega \widetilde{A}_{r k}\left(r_{0}\right),
\end{aligned}
$$

where

$$
\widetilde{A}_{r k}\left(r_{0}\right)=\frac{z_{\infty}}{z_{0}} \sum_{m} b_{k m} A_{r m}\left(r_{0}\right) .
$$

Note that the boundary condition for the derivative of scalar potential (44) is a result of zero radial component of current density at $r=r_{0}$.

In general, (41) can be resolved through the integrals similarly to (14)

$$
\begin{aligned}
& V_{k}=G_{k} I_{1}\left(\eta_{k} r\right)+i \omega I_{1}\left(\eta_{k} r\right) \int_{r}^{r_{0}} q_{k}\left(r^{\prime}\right) K_{1}\left(\eta_{k} r^{\prime}\right) r^{\prime} d r^{\prime}+ \\
& i \omega K_{1}\left(\eta_{k} r\right) \int_{0}^{r} q_{k}\left(r^{\prime}\right) I_{1}\left(\eta_{k} r^{\prime}\right) r^{\prime} d r^{\prime}
\end{aligned}
$$

where the coefficients $G_{k}$ are determined from the boundary condition (43) as:

$$
G_{k}=-\frac{i \omega}{\eta_{k} I_{1}^{\prime}\left(\eta_{k} r_{0}\right)}\left(\widetilde{A}_{r m}\left(r_{0}\right)+K_{1}^{\prime}\left(\eta_{k} r_{0}\right) \int_{0}^{r_{0}} q_{k} I_{1}\left(\eta_{k} r^{\prime}\right) r^{\prime} d r^{\prime}\right),
$$

where $I_{1}^{\prime}, K_{1}^{\prime}$ are the first derivatives of Bessel functions. The scalar potential derivative at $z= \pm z_{0}$ can be expressed through $\sigma^{-1} \operatorname{div} \sigma \vec{A}$. Indeed (4) can be rewritten as:

$$
\nabla^{2} V=-\frac{i \omega}{\sigma} \operatorname{div} \sigma \vec{A} .
$$

The divergence in the right part of (47) is not zero anymore if we take the ends of cylinder into consideration. For the Coulomb gauge $\operatorname{div} \sigma \vec{A}$ is zero everywhere inside the cylinder but the surface where it has a jump of $\sigma(\vec{A} \vec{n})$ that is called as a surface divergence [18]. Because the conductivity is discontinuous at $z= \pm z_{0}$ and the integration totally includes the jump of $\sigma$ we have

$$
\begin{aligned}
& \frac{1}{z_{0}} \int_{-z_{0}}^{z_{0}} \operatorname{div}(\sigma \vec{A}) \sin \eta_{k} z d z=\frac{1}{z_{0}} \int_{-z_{0}}^{z_{0}} A_{z} \frac{\partial \sigma}{\partial z} \sin \eta_{k} z d z=. \\
& \frac{2(1)^{k+1}}{z_{0}} \sigma A_{z}\left(z_{0}\right)
\end{aligned}
$$

Thus the right part in (41) can be replaced by the integral of $\operatorname{div} \sigma \vec{A}$ after accounting for the angular dependence of vector potential components (22)-(24)

$q_{k}=\frac{-i \omega}{z_{0} \sigma} \int_{-z_{0}}^{z_{0}}\left[\frac{\partial\left(\sigma \mathrm{A}_{r}\right)}{\partial r}+\frac{\partial\left(\sigma \mathrm{A}_{z}\right)}{\partial z}+\frac{\sigma\left(\mathrm{A}_{r}-\mathrm{A}_{\phi}\right)}{r}\right] \sin \eta_{k} z d z$.

After replacing the vector potential components by harmonics over the axial coordinate and subsequent integration we obtain

$$
q_{k}=-i \omega\left(\frac{\partial \tilde{A}_{r k}}{\partial r}-\frac{\tilde{A}_{\phi-r, k}}{r}-\eta_{k} \widetilde{A}_{z k}\right),
$$

where

$$
\tilde{A}_{\phi-r, k}=\frac{z_{\infty}}{z_{0}} \sum_{m} b_{m k} A_{\phi-r, m}, \tilde{A}_{z k}=\frac{z_{\infty}}{z_{0}} \sum_{m} a_{m k} A_{z m} .
$$

The coefficients $\widetilde{A}_{z k}$ are the result of fitting the axial component $A_{z}$ to the cosine harmonics along the cylinder 
length. We use values of potential up to the ends of the interval $\left[-z_{0}, z_{0}\right]$ where $-i \sigma \omega A_{z}$ is zeroed because the jump in the conductivity. This allows us to account for the surface divergence at $z= \pm z_{0}$. The computation of $q_{k}$ using (50) is more complex than using a straight expression (42). However including $\operatorname{div} \sigma \vec{A}$ in the equation for the scalar potential enforces the divergence-free condition (4). To combine the advantages of two methods for computation of $q_{k}$, one can add $\operatorname{div} \vec{A}(=0)$ to the jump of potential in expression (42)

$q_{k}=(-1)^{k} \frac{2 i \omega}{z_{0}} \mathrm{~A}_{z}\left(z_{0}\right)+i \omega \frac{z_{\infty}}{z_{0}} \sum_{l}\left(\frac{\partial A_{r l}}{\partial r}-\frac{A_{\phi-r, l}}{r}-\lambda_{l} A_{z l}\right) b_{k l}$,

The latter expression for $q_{k}$ accurately includes the surface divergence and enforces (4) at the same time.

The harmonics of current density can be expressed through the harmonics of potentials as follows:

$$
\begin{aligned}
& j_{r k}=-\sigma\left(i \omega \widetilde{A}_{r k}+\frac{\partial V_{k}}{\partial r}\right), \\
& j_{\phi k}=-\sigma\left(i \omega \widetilde{A}_{\phi k}+\frac{V_{k}}{r}\right), \\
& j_{z k}=-\sigma\left(i \omega \widetilde{A}_{z k}+\eta_{k} V_{k}\right)
\end{aligned}
$$

The solutions (32),(39),(40) and (45) contain the unknown coefficients of harmonics $A_{r+\phi, m} A_{\phi p r, \mathrm{~m},}, A_{z m}, V_{k}$. After approximating the integrals by a high accuracy quadratures on the grid $r_{n}=r_{n-1+1} h_{r n}, n=1, N$ built for the interval $\left[0, r_{0}\right]$, these expressions form a system of linear equations of order of $4 N M$ where $M$ is the number of harmonics in series (22)-(24),(28). The structure of the system is similar to (15) but it is more complex since the current density components in (32), (39), (40) should be expressed through the vector and scalar potential harmonics using (52). To simplify the solution, the system of equations can be solved also using the iterative approach. In this case the current density (52) is split so that the parts associated with the time derivatives of vector potential are included into the matrix coefficients while the terms associated with the electric potential are kept in the right part of system.

The analytical method has been applied to the copper cylinder of radius, $r_{0}$ of $1 \mathrm{~cm}$ and half length, $z_{0}$ of $1 \mathrm{~cm}$. The number of harmonics $M$ has been selected in the range $\geq 30$, and the far field boundary conditions have been applied at $\mathrm{z} \geq 3 \mathrm{z}_{0}$. The distribution of current density is presented in Fig. 8-10 in comparison with the results from FEA [17] and FDM [19]. A pure hexahedral mesh in all directions has been used in both numerical methods. The mesh has been refined in both methods until they provided the close solutions in terms of local fields, energy and moment of induced currents. For FDM, the symmetry over the polar angle (22)-(24) has been used first, and then four coupled 2$\mathrm{D}$ equations have been solved. 2-D equations have been approximated using the integral-interpolation method [19]. The finite-differential schemes for the equations are similar to those reported in [20].

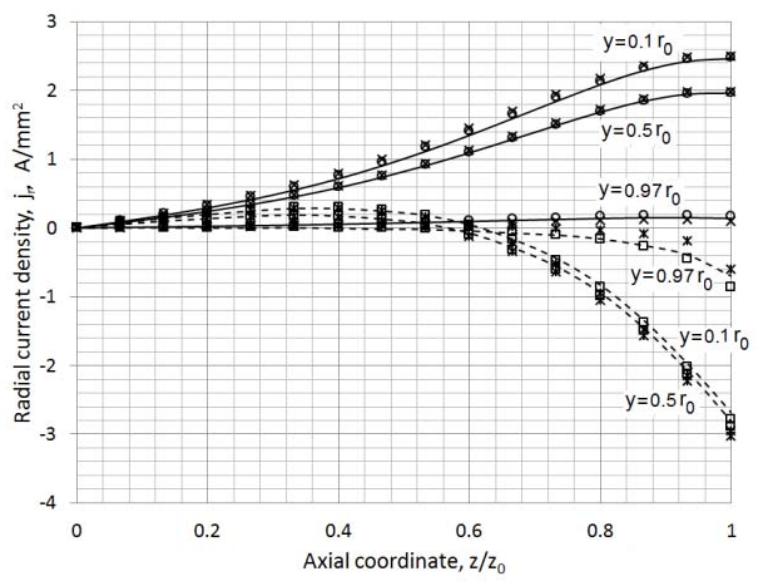

Figure 8: Radial component of current density in $Y Z$-plane across the cylinder of $r_{0}=1 \mathrm{~cm}, z_{0}=1 \mathrm{~cm}$ at $f=300 \mathrm{~Hz}, B_{0}=10$ $\mathrm{mT}$ : solid line $=$ real $j_{\mathrm{r}}$, analytics, dash line $=$ imaginary $j_{\mathrm{r}}$, analytics; o $=$ real $j_{\mathrm{r}}$, FEA, $\square=$ imaginary $j_{\mathrm{r}}$, FEA; $x=\operatorname{real} j_{\mathrm{r}}$ , FDM, $*=$ imaginary $j_{\mathrm{r}}$, FDM.

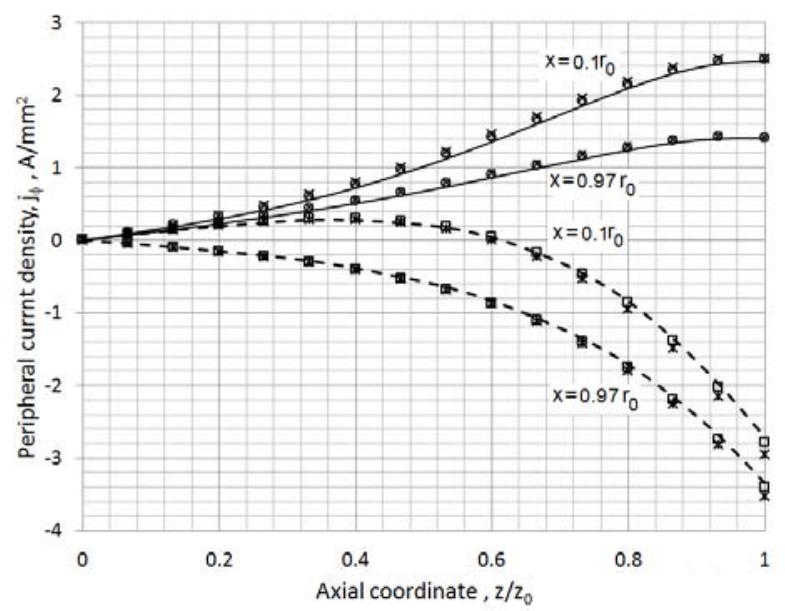

Figure 9: Peripheral component of current density in $X Z$ plane across the cylinder of $r_{0}=1 \mathrm{~cm}, z_{0}=1 \mathrm{~cm}$ at $f=300 \mathrm{~Hz}$, $B_{0}=10 \mathrm{mT}$, data labels are the same as in Fig. 8 .

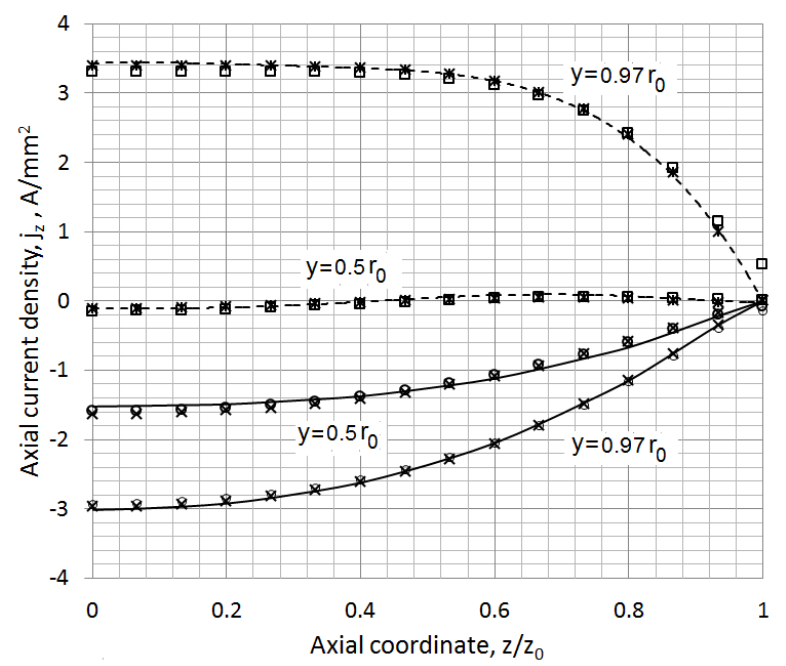

Figure 10: Axial component of current density in $Y Z$-plane across the cylinder of $r_{0}=1 \mathrm{~cm}, z_{0}=1 \mathrm{~cm}$ at $f=300 \mathrm{~Hz}, B_{0}=10$ $\mathrm{mT}$, data labels are the same as in Fig. 8. 


\subsection{Hollow cylinder}

Similarly to the cylinder in the axial field the calculation formulas for the transverse field can be applied in the case of the hollow cylinder of the inner radius $r_{i}$, outer radius $r_{0}$ and axial length $2 z_{0}$. The equations for the components of vector potential (32),(39), and (40) differ only by the integration range. The lower zero limits in integrals (32),(39) and (40) should be replaced by $r_{i}$. Thus the grid along the radial coordinate is built in the interval $\left[r_{i}, r_{0}\right]$. The expression for the scalar electric potential contains an additional Bessel function of second kind $K_{l}\left(\eta_{k} r\right)$ with the constant coefficients

$$
\begin{aligned}
& V_{k}=G_{k} I_{1}\left(\eta_{k} r\right)+F_{k} K_{1}\left(\eta_{k} r\right)+I_{1}\left(\eta_{k} r\right) \\
& \times \int_{r}^{r_{0}} q_{k}\left(r^{\prime}\right) K_{1}\left(\eta_{k} r^{\prime}\right) r^{\prime} d r^{\prime}+K_{1}\left(\eta_{k} r\right) \int_{r_{i}}^{r} q_{k}\left(r^{\prime}\right) I_{1}\left(\eta_{k} r^{\prime}\right) r^{\prime} d r^{\prime}
\end{aligned}
$$

This is because the scalar potential is considered for $r>0$. Thus the solution (53) that includes infinite functions $K_{l}\left(\eta_{k} r\right)$ at $r=0$ is finite everywhere in the hollow cylinder. The boundary condition on the outer surface of the hollow cylinder is the same as (44). Similarly, on the inner surface we have

$$
{\frac{\partial V_{k}}{\partial r}}_{\mid r=r_{i}}=-i \omega \widetilde{A}_{k r}\left(r_{i}\right) .
$$

The coefficients $G_{k}$ and $F_{k}$ are determined from the boundary condition system (44) and (54) as

$$
\begin{aligned}
& G_{k}=\left(K_{k}^{\prime}\left(\eta_{k} r_{0}\right) g_{k}-K_{k}^{\prime}\left(\eta_{k} r_{i}\right) f_{k}\right) / D_{k}, \\
& F_{k}=\left(I_{k}^{\prime}\left(\eta_{k} r_{0}\right) f_{k}-I_{k}^{\prime}\left(\eta_{k} r_{i}\right) g_{k}\right) / D_{k},
\end{aligned}
$$

where

$$
\begin{aligned}
& g_{k}=-i \omega \widetilde{A}_{r k}\left(r_{0}\right) / \eta_{k}-K_{1}^{\prime}\left(\eta_{k} r_{0}\right) \int_{r_{i}}^{r_{0}} q_{k} I_{1}\left(\eta_{k} r^{\prime}\right) r^{\prime} d r^{\prime} \\
& f_{k}=-i \omega \widetilde{A}_{r k}\left(r_{i}\right) / \eta_{k}-I_{1}^{\prime}\left(\eta_{k} r_{i}\right) \int_{r_{i}}^{r_{0}} q_{k} K_{1}\left(\eta_{k} r^{\prime}\right) r^{\prime} d r^{\prime} \\
& D_{k}=I_{k}^{\prime}\left(\eta_{k} r_{0}\right) K_{k}^{\prime}\left(\eta_{k} r_{i}\right)-I_{k}^{\prime}\left(\eta_{k} r_{i}\right) K_{k}^{\prime}\left(\eta_{k} r_{0}\right) .
\end{aligned}
$$

The harmonics of $A_{z}$ in the bore of the cylinder

$$
\left.A_{z m}\right|_{r \leq r_{i}}=-B_{0} r d_{m 0}+\mu_{0} I_{1}\left(\lambda_{m} r\right) \int_{r_{i}}^{r_{0}} \widetilde{j}_{z m}\left(r^{\prime}\right) K_{1}\left(\lambda_{m} r^{\prime}\right) r^{\prime} d r^{\prime}
$$

For $A_{\phi}$ one can derive

$$
\begin{aligned}
& \left.A_{\phi m}\right|_{r \leq r_{i}}=\frac{\mu_{0}}{2} I_{0}\left(\lambda_{m} r\right) \int_{r_{i}}^{r_{0}}\left[\widetilde{j}_{\phi m}\left(r^{\prime}\right)+\widetilde{j}_{r m}\left(r^{\prime}\right)\right] K_{0}\left(\lambda_{m} r^{\prime}\right) r^{\prime} d r^{\prime}, \\
& +\frac{\mu_{0}}{2} I_{2}\left(\lambda_{m} r\right) \int_{r_{i}}^{r_{0}}\left[\widetilde{j}_{\phi m}\left(r^{\prime}\right)-\widetilde{j}_{r m}\left(r^{\prime}\right)\right] K_{2}\left(\lambda_{m} r^{\prime}\right) r^{\prime} d r^{\prime} .
\end{aligned}
$$

The component $B_{x}$ on $z$-axis is

$$
B_{x}=\frac{1}{r} \frac{\partial A_{z}}{\partial \phi}-\frac{\partial A_{\phi}}{\partial z}
$$

Combining (56)-(58) we obtain the flux density on the axis of the cylinder

$$
\begin{aligned}
& \left.B_{x}\right|_{x ; y=0}=-B_{0}+\frac{\mu_{0}}{2} \sum_{m} \lambda_{m} \cos \left(\lambda_{m} z\right)\left[\int_{r_{i}}^{r_{0}} \widetilde{j}_{z m}\left(r^{\prime}\right) K_{1}\left(\lambda_{m} r^{\prime}\right) r^{\prime} d r^{\prime},\right. \\
& \left.-\int_{r_{i}}^{r_{0}}\left(\widetilde{j}_{r m}\left(r^{\prime}\right)+\widetilde{j}_{\phi m}\left(r^{\prime}\right)\right) K_{0}\left(\lambda_{m} r^{\prime}\right) r^{\prime} d r^{\prime}\right] .
\end{aligned}
$$

Other components are zero on the axis of the cylinder, thus $B_{x}$ gives us the magnitude of the field on $z$-axis.

Similarly to the solid cylinder the formulas for the hollow cylinder have been verified by the comparison with the induced currents computed by numerical methods (Fig.1114) in the range of frequencies. The steps in the radial grid have been selected based on the frequency of the applied field. For example, at least 30 steps $(N=31)$ over the radial coordinate is used for the frequency of $2 \mathrm{kHz}$ in the case study with the results presented in Fig. 14.

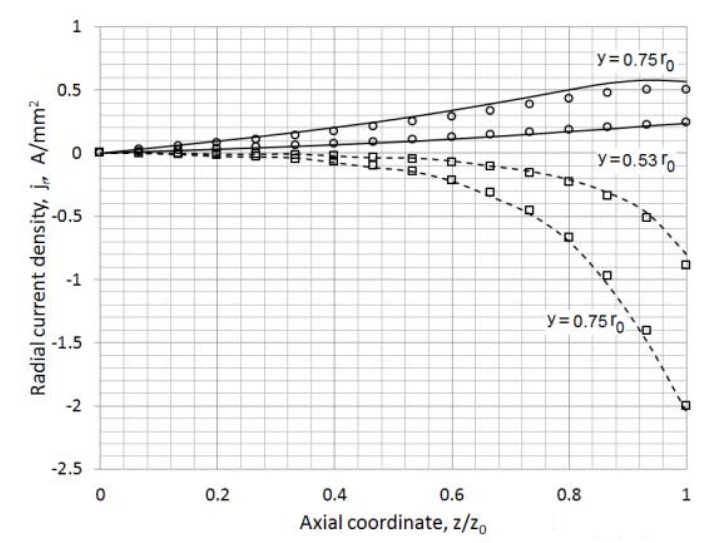

Figure 11: Radial component of current density in $Y Z$ section of a hollow cylinder of $r_{0}=1 \mathrm{~cm}, r_{i}=0.5 \mathrm{~cm} z_{0}=1$ at $f=300 \mathrm{~Hz}, B_{0}=10 \mathrm{mT}$ : solid line $=$ real $j_{\mathrm{z}}$, analytics, dash line =imaginary $j_{\mathrm{z}}$, analytics; o $=$ real $j_{\mathrm{z}}, \mathrm{FEA}, \square=$ imaginary $j_{\mathrm{z}}$, FEA.

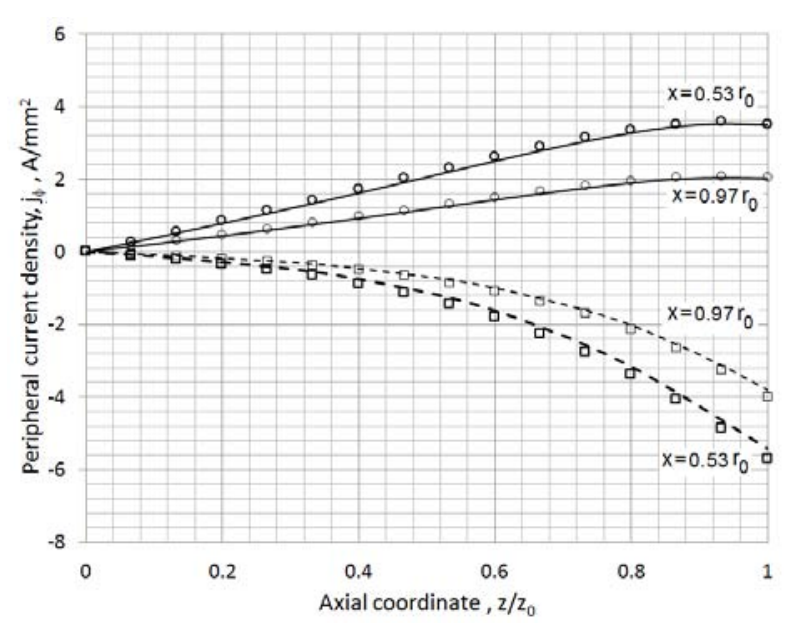

Figure 12: Peripheral component of current density in $X Z$ section of a hollow cylinder of $r_{0}=1 \mathrm{~cm}, r_{i}=0.5 \mathrm{~cm}, z_{0}=1 \mathrm{~cm}$ at $f=300 \mathrm{~Hz}, B_{0}=10 \mathrm{mT}$, data labels are the same as in Fig. 11 . 


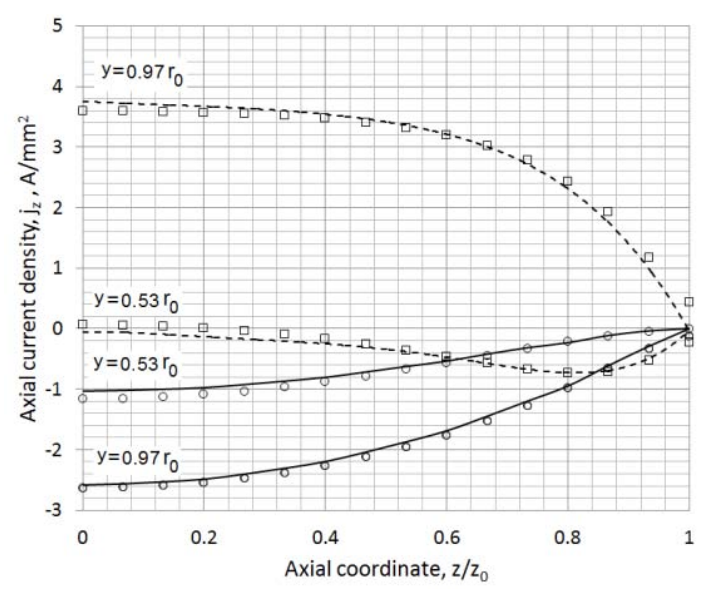

Figure 13: Axial component of current density in $Y Z$-section of a hollow cylinder of $r_{0}=1 \mathrm{~cm}, r_{i}=0.5 \mathrm{~cm}, z_{0}=1$ at $f=300$ $\mathrm{Hz}, B_{0}=10 \mathrm{mT}$ at $f=300 \mathrm{~Hz}, B_{0}=10 \mathrm{mT}$, data labels are the same as in Fig. 11.

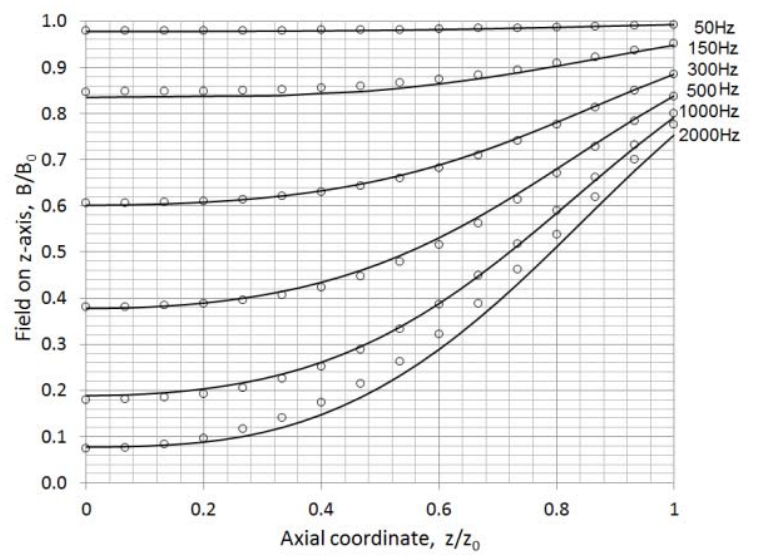

Figure 14: Field on axis of the hollow cylinder of $r_{0}=1 \mathrm{~cm}$, $r_{i}=0.5 \mathrm{~cm} z_{0}=1 \mathrm{~cm}$ at different frequencies: solid line $=$ analytics, o $=$ FEA.

\subsection{Cylindrical shell}

A cylindrical shell is a special case of the hollow cylinder when we can neglect a radial dependence of current density[2]. First, consider how the thin shell approximation can simplify the solution in case of hollow cylinder. Since the thickness of the shell typically is significantly less than its radius it is practical to consider a one-layer $(N=1)$ approximation for the derived solution. Indeed if we neglect the dependence of electric scalar on the radial coordinate, from (41) we can derive

$$
V_{k}\left(r_{0}\right)=-\frac{q_{k}}{1 / r_{0}^{2}+\eta_{k}^{2}}
$$

This simple approach has few limits. A one-layer approximation totally ignores the difference in the current density across the shell thickness although it accounts for the radial component of vector potential, and hence for the radial current density associated with its time derivative. Also the one-layer model may lead to the ill-defined matrix

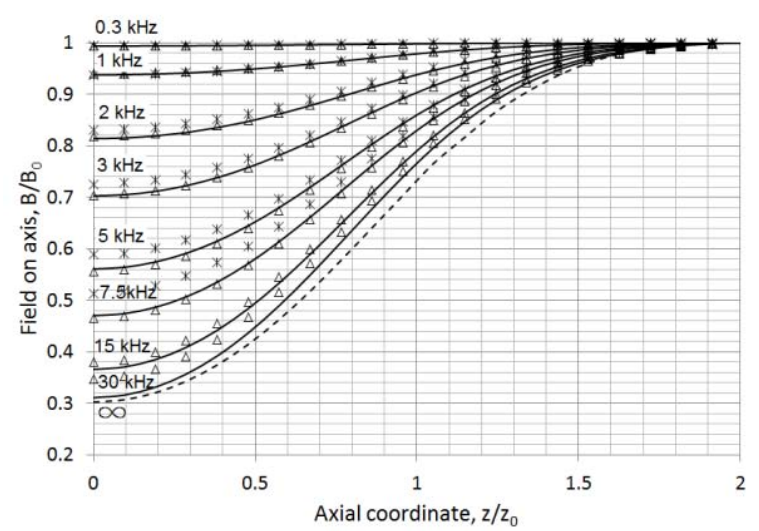

Figure 15: Field on axis of the cylindrical shell of $r_{0}=1 \mathrm{~cm}$, $d_{r}=0.35 \mathrm{~mm}, z_{0}=1 \mathrm{~cm}$ at different frequencies: solid line $=$ 5 steps in radial grid , $*=V_{k}$ calculated per (60), $\Delta=$ scalar magnetic potential method per (74), dash line = superconducting shell.

for the harmonics of vector potential starting at high frequencies. It is illustrated in Fig. 15 for a $0.35 \mathrm{~mm}$ thick copper shell of $1 \mathrm{~cm}$ radius. When the frequencies are low the field on the axis is in a good agreement with the accurate approximation of the shell by five layers $(N=6)$. When the frequency increases the matrix condition number becomes too large, and the approximation (60) at $N=1$ starts to give us an inaccurate field on the axis. It should be noted that the calculation of $q_{k}$ in (60) using a straight expression (41) gives a more accurate result since in case of just one layer the derivative $d A_{r} / d r$ in the expression for $\operatorname{div} \vec{A}$ is hard to determine accurately.

The alternative solution for the thin shell can be derived using a concept of stream function [2] and a scalar magnetic potential [21-22]. The magnetic field is split into the applied field, $\vec{H}_{0}$ and the field from the eddy currents, $\vec{H}_{e}$ where the normal component of $x$-oriented field on the shell surface is $H_{0 r}=H_{0} \cos \phi$. Outside the cylindrical shell we can use the scalar magnetic potential $U$ to calculate the field from the eddy currents $\vec{H}_{e}=-\nabla U$. The magnetic scalar potential satisfies the Laplace equation everywhere but the shell. The whole space along the radial coordinate is divided by the shell into two regions: $r>r_{0}$ (marked by upper index + ) and $r<r_{0}$ (marked by upper index -). The scalar potential inside $(-)$ and outside $(+)$ the shell can be written in terms of Bessel functions as

$$
\begin{aligned}
& U^{-}=\cos \phi \sum_{m=1}^{M} C_{m}^{-} I_{1}\left(\lambda_{m} r\right) \cos \lambda_{m} z, \\
& U^{+}=\cos \phi \sum_{m=1}^{M} C_{m}^{+} K_{1}\left(\lambda_{m} r\right) \cos \lambda_{m} z
\end{aligned}
$$

where $C_{m}{ }^{-}$and $C_{m}{ }^{+}$are the constants. Because the normal component of the field is continuous everywhere including the shell surface the constants in (61) relate as:

$$
C_{m}^{-} I_{1}^{\prime}\left(\lambda_{m} r_{0}\right)=C_{m}^{+} K_{1}^{\prime}\left(\lambda_{m} r_{0}\right) .
$$


The field tangential component has a jump by the amount of surface current density $\vec{j}_{s}=h_{r} \vec{j}$ when crossing the shell thickness from inside to outside

$$
\vec{j}_{s} \underset{\substack{r=r_{0} \\ 0 \leq z \leq z_{0}}}{ }=\vec{n} \times\left(\vec{H}_{e}^{+}-\vec{H}_{e}^{-}\right) .
$$

The scalar magnetic potential has also a jump [18] on the shell surface $u=U^{+}-U$. After expressing the field through the scalar magnetic potential in (63) one can write

$$
\left.\vec{j}_{s}\right|_{\substack{r=r_{0} \\ 0 \leq z \leq z_{0}}}=\vec{n} \times\left(\nabla_{s} u\right),
$$

where index $s$ means that the nabla operator $\nabla_{\mathrm{s}}$ works on the surface, $\vec{n}$ is the unit vector normal to the surface. In terms of components (64) can be rewritten as

$$
j_{s \phi}=\frac{\partial u}{\partial z}, \quad j_{s z}=-\frac{1}{r_{0}} \frac{\partial u}{\partial \phi} .
$$

In accordance with [2] the stream function $\Psi(\phi, z)$ relates to the current density as

$$
\vec{j}_{s}=h_{r} \sigma \nabla_{s} \times \Psi \vec{n},
$$

or in terms of components

$$
j_{s \phi}=h_{r} \sigma \frac{\partial \Psi}{\partial z}, j_{s z}=-h_{r} \sigma \frac{1}{r_{0}} \frac{\partial \Psi}{\partial \phi} .
$$

The stream function satisfies the equation

$$
\frac{1}{r_{0}^{2}} \frac{\partial^{2} \Psi}{\partial \phi^{2}}+\frac{\partial^{2} \Psi}{\partial z^{2}}=i \omega \mu_{0} H_{r}
$$

where $H_{r}$ is the radial, i.e. component of the field normal to the cylindrical surface. Comparing (65) with (67) we can conclude that the potential jump $u$ satisfies the equation similar to (68), namely

$$
\frac{1}{r_{0}^{2}} \frac{\partial^{2} u}{\partial \phi^{2}}+\frac{\partial^{2} u}{\partial z^{2}}=i \omega \mu_{0} \sigma h_{r} H_{r}
$$

The potential jump $u$ exists only at $r=r_{0}$ and $0 \leq z \leq z_{0}$ and it can be presented it in the form of series

$$
u=\cos \phi \sum_{m=1}^{M} u_{m} \cos \eta_{m} z
$$

After substitution (70) in (69) and integrating over $\left[0, z_{0}\right]$ the coefficients $u_{m}$ are determined as follows:

$$
u_{m}=-\frac{i \omega \mu_{0} \sigma h_{r}}{\left(\eta_{m}^{2}+1 / r_{0}^{2}\right)} \frac{z_{\text {inf }}}{z_{0}}\left\lfloor d_{0 m}+\sum_{l} \lambda_{l} b_{l m} C_{l}^{-} I_{1}\left(\lambda_{l} r_{0}\right)\right\rfloor .
$$

The second equation for the coefficients $C_{m}{ }^{-}$and $u_{m}$ can be determined from the potential junction at $r=r_{0}$

$$
U^{-}=\begin{aligned}
& U^{+}-u, 0 \leq z \leq z_{0} \\
& U^{+}, z_{0} \leq z \leq z_{\mathrm{inf}}
\end{aligned}
$$

After integration (72) over $\left[0, z_{0}\right]$ and substitution $C_{m}{ }^{+}$ from (62) we obtain

$$
C_{m}^{-}=\left[I_{1}\left(\lambda_{m} r_{0}\right)-K_{1}\left(\lambda_{m} r_{0}\right) \frac{I_{1}^{\prime}\left(\lambda_{m} r_{0}\right)}{K_{1}^{\prime}\left(\lambda_{m} r_{0}\right)}\right]^{-1} \sum_{l} b_{m l} u_{l} .
$$

Equations (71),(73) form the system of linear equations for the coefficients $C_{m}{ }^{-}$and $u_{m}$ that can be solved directly. However (71),(73) can be solved by iterations with some under relaxation factor. We assume $C_{m}{ }^{-}=0$ for the first iteration, find $u_{m}$ from (71) and update $C_{m}{ }^{-}$using (73) with under relaxation. We iterate (71) and (73) until the convergence tolerance is achieved. The iterative method implements the perturbation of the normal field $H_{r}$ by the induced field starting with $H_{0}$ as a first approximation for the field on the shell surface. The iterative algorithm gives a more steady solution at high frequencies when the matrix of system (71),(73) becomes ill defined .

The magnetic field on $z$-axis is calculated as

$$
\left.B_{x}\right|_{x ; y=0}=-B_{0}-\frac{1}{2} \sum_{m} \lambda_{m} C_{m}^{-} \cos \left(\lambda_{m} z\right) .
$$

The thin sheet approximation using the scalar potential works well even at high frequencies (Fig.15). The discrepancy from the accurate solution $(\mathrm{N}=6)$ increases at frequencies above $15 \mathrm{kHz}$ when the shielding capacity of the shell is close to saturation. The solution $(71),(73)$ becomes unresponsive to the frequency because of difficulties in the accurate estimation of the normal component of the resultant field $H_{r}$ on the shell surface (right part of (69)).

\subsection{Magnetic moment and power loss}

The magnetic moment $M_{x}$ of currents induced in the cylinder is

$$
M_{x}=8 \int_{0}^{\pi / 2} \int_{r_{i}}^{r_{0}} \int_{0}^{z_{0}}\left(j_{z} r \sin \phi+j_{y} z\right)(r d \phi) d r d z
$$

After substituting series (25)-(27) and integrating one can find

$$
\begin{aligned}
M_{x}= & 2 \pi \sum_{m=1}^{M}(-1)^{(m+1)}\left\lfloor\frac{1}{\eta_{m}} \sum_{n=1}^{N} j_{z m}\left(r_{n}\right) r_{n}^{2} w_{n} h_{r n}\right. \\
& \left.+\frac{1}{\eta_{m}^{2}} \sum_{n=1}^{N}\left(j_{r m}\left(r_{n}\right)+j_{\phi m}\left(r_{n}\right)\right) r_{n} w_{n} h_{r n}\right] .
\end{aligned}
$$

The power loss in the cylinder is

$$
\begin{aligned}
& P=\frac{1}{2 \sigma} \int_{0}^{2 \pi r_{0}} \int_{r_{i}}^{z_{0}} \int_{0}\left(j_{r} j_{r}^{*}+j_{\phi} j_{\phi}^{*}+j_{z} j_{z}^{*}\right) r d z d r= \\
& \frac{\pi z_{0}}{2 \sigma} \sum_{n=1}^{N} \sum_{m=1}^{M} \sum_{l=1}^{M}\left[c_{m l}^{-}\left(j_{r m} j_{r l}^{*}+j_{\phi m} j_{\phi l}^{*}\right)+c_{m l}^{+} j_{z m} j_{z l}^{*}\right] w_{n} r_{n} \Delta_{r n},
\end{aligned}
$$

where

$$
c_{m l}^{ \pm}=\frac{1}{z_{0}}\left(\frac{\sin \left(\eta_{m}-\eta_{l}\right) z_{0}}{\eta_{m}-\eta_{l}} \pm \frac{\sin \left(\eta_{m}+\eta_{l}\right) z_{0}}{\eta_{m}+\eta_{l}}\right) .
$$

The results of calculation of power loss and magnetic moment of the copper solid cylinder using (75a)-(76) in test models agree well with the data from FEA and FDM (Fig.16). 


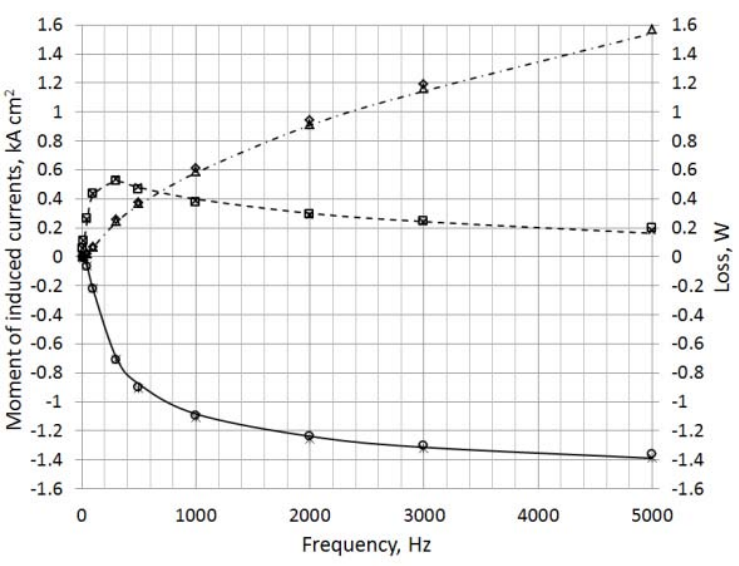

Figure 16: Moment of current and power loss in the solid cylinder of $r_{0}=1 \mathrm{~cm}, z_{0}=1 \mathrm{~cm}$ at $B_{0}=10 \mathrm{mT}$ of transverse field: solid line $=$ real $M$, analytics, dash line =imaginary $M$, analytics; o = real $M$, FEA, $\square=$ imaginary $M$, FEA; $\times=$ real $M, \mathrm{FDM}, *=$ imaginary $M, \mathrm{FDM}$ dash-dot line = power loss, analytics; $\Delta=$ power loss, FEA; $\diamond=$ power loss, FDM

\section{Conclusions}

The analytical solutions for the eddy current problem in the conductive solid and hollow cylinders in cases of axial and transverse fields have been derived. The magnetic field outside the cylinder or inside the bore is expressed in terms of Bessel functions. Formulas have been verified by the comparison of the calculation results in test models with the FDM method and modern FEA codes. Formulas for the power loss and magnetic moment of currents induced in the cylinder have been also found. These have been validated over a wide range of frequencies within the limits of quasistatic approximation.

\section{References}

[1] J. Lameraner and M. Štafl, Eddy currents. London: Iliffe Books Ltd., 1966.

[2] H. E. Knoepfel, Magnetic fields. New York-Toronto: John Wiley \&Sons,Inc., 2000.

[3] V. V. Batygin and I. N. Toptygin, Problems in Electrodynamics, London,U.K.: Academic, problems 368 and 371, 1976.

[4] R. Grimberg, E. Radu, O. Mihalache, A. Savin, Calculation of the induced electromagnetic field created by an arbitrary current distribution located outside a conductive cylinder, J. Phys. D: Appl. Phys. 30228530 pp.2285-2291,1997.

[5] E. H. Brandt, Superconductor disks and cylinders in an axial magnetic field. I. Flux penetration and magnetization curves, Phys. Rev. B, vol. 58, no. 10, pp. 6506-6522, 1998.

[6] H. S. Lopez, M. Poole, S. Crozier, Eddy current simulation in thick cylinders of finite length induced by coils of arbitrary geometry, Journal of Magnetic Resonance. 207 ,pp.251-261, 2010.
[7] J. R. Bowler and T. P. Theodoulidis, Eddy currents induced in a conducting rod of finite length by a coaxial encircling coil, J. Phys. D: Appl. Phys. 38, pp. 28612868, 2005.

[8] M. Perry, T. Jones, Eddy current induction in a solid conducting cylinder with a transverse magnetic field, IEEE Trans. on Magn., vol. 14, No 4, pp.227231,1978.

[9] T.H. Fawzi, K .F. Ali, P. E. Burke, Eddy current losses in finite length conducting cylinders, IEEE Trans. on Magn. vol.19 No 5, pp.:2216-2218, 1983.

[10] T. Morisue, M. Fukumi, 3-D eddy current calculation using the magnetic vector potential, IEEE Trans. on Magn., vol. 24, No. 1 , pp.106-109, 1988.

[11] Q. S. Huang, L. Krahenbuhl, A. Nicolas, Numerical calculation of steady-state skin effect problems in axisymmetry, IEEE Trans. on Magn., vol. 24, No. 1 , pp.201-204, 1988.

[12] L. R. Turner et al ,Results from the FELIX experiments on electromagnetic effects of hollow cylinders", IEEE Trans. on Magn., vol. 21, No. 6, pp.2324-2328, 1985.

[13] International Electromagnetic Workshops: Test Problems, April 1986. Available online: https://www.osti.gov/scitech/servlets/purl/7179128

[14] R. P. Feynman, Feynman lectures on physics. Volume 2: Mainly electromagnetism and matter. Reading, MA.: Addison-Wesley, 1964.

[15] G. A. Grinberg, The Selected Problems of Mathematical Theory of Electric and Magnetic Phenomena. MoscowLeningrad, Russia: Acad Sci USSR, 1948.

[16] W. Rosenheinrich, Tables of some indefinite integral of Bessel functions of integer order, 2017. Available online:

http://web.eah-jena.de/ rsh/Forschung/Stoer/besint.pdf

[17] Opera 2D, User Guide and Opera 3D, User Guide. Cobham Technical Services, Vector Fields Software, Kidlington, UK, Mar. 2016.

[18]I.E. Tamm, Fundamentals of the theory of electricity, Moscow : Mir Publishers, 1979.

[19] A. A. Samarskiy, Theory of Finite Difference Schemes, Moscow: Nauka, 1977.

[20] Y. Zhilichev, Superconducting cylinder of finite length in transverse magnetic field, Latvian Journal of Physics and Technical Sciences, No5, pp.14-21, 2001.

[21]B. Ancelle, A. Nicolas, and J. C. Sabonnadiere, A boundary integral equation method for high frequency eddy currents, IEEE Trans. on Magn., vol. 17, No. 6 , pp.2568-2570, 1981.

[22]J. Poltz and K. Romanowski, Solution of quasistationary fields problems by means of magnetic scalar potential, IEEE Trans. on Magn., vol. 19, No. 6 , pp.2425-2428, 1983.

[23]M. Filtz and H. Bussing, Screening attenuation of conducting and ferromagnetic hollow cylinders of finite length, Electrical Engineering, vol. 90, pp.469-478, 2008 . 